\title{
APLICACIÓN AMBIENTAL DEL MÉTODO DE RESISTIVIDAD ELÉCTRICA EN EL MODELADO DEL ACUÍFERO COSTERO EN PLAYA PANAMÁ, GUANACASTE, COSTA RICA
}

\author{
Daniel Murillo Montoya \\ Departamento de Geologia, Universidade Federal do Paraná, Curitiba, Brasil. \\ Apdo. 1602-1100, Tibás, Costa Rica \\ danimm@costarricense.cr
}

(Recibido: 12/03/07; aceptado 03/05/08)

\begin{abstract}
The conceptual model of the coastal aquifer of Playa Panamá was complemented by new resistivity measurements. Twelve Vertical Electric Soundings (VESs) were carried out on the Quaternary sediments, forming the hydric reservoir. The geoelectric data were inverted for a conceptual model of four hydrogeologic layers: 1) Topsoil, 2) the non saturated zone, 3) the saturated zone and 4) the regional geologic basement, represented by the Nicoya Complex. The results were correlated with stratigraphic and hydrogeological data from wells. The geophysical method used allowed to define new values of the sediments thickness, and also helped to identify the saline dispersion zone. The isopachs map suggests a decrease in the aquifer's thickness towards the sea, with values between 13 and $27 \mathrm{~m}$.

Key words: Applied geophysics, electric resistivity, environmental geology, hydrogeology, coastal aquifer, Playa Panamá, Costa Rica.

RESUMEN: El modelo conceptual del acuífero costero de Playa Panamá fue complementado por medio de la utilización del método de resistividad eléctrica con corriente continua. Fueron efectuados 12 Sondeos Eléctricos Verticales (SEVs) con el arreglo electródico Schlumberger sobre los sedimentos recientes que conforman el almacén hídrico. Los datos de resistividad aparente fueron procesados usando la técnica de inversión, partiendo de un modelo general de cuatro capas: 1) suelo superficial, 2) zona no saturada, 3) zona saturada y 4) basamento regional, este último constituido por el Complejo de Nicoya. Los resultados de este proceso fueron correlacionados con datos litoestrátigraficos de pozos en el área de estudio. El método geofísico utilizado permitió establecer datos nuevos de los espesores de los sedimentos y ayudó a identificar la presencia de la zona de dispersión salina en el subsuelo. El mapa de isopacas refleja la disminución del espesor del acuífero en dirección al mar, el cual exhibió valores entre 13 y 27 m.

Palabras clave: Geofísica aplicada, resistividad eléctrica, geología ambiental, hidrogeología, acuífero costero, Playa Panamá, Costa Rica.
\end{abstract}




\section{INTRODUCCIÓN}

Varios acuíferos costeros en la Península de Nicoya son explotados de manera poco sustentable. Arellano \& Vargas (2001) y Losilla (1993) advierten sobre los problemas de contaminación salina que presentan al menos nueve de las veinticinco zonas acuíferas identificadas por estos autores en esa región.

Las características climáticas de la Península de Nicoya, así como las políticas económicas y las actividades ambientales que se realizan sobre sus zonas acuíferas, particularmente el desarrollo acelerado de infraestructura turística, han puesto en amenaza a la calidad fisicoquímica del agua subterránea y la continuación de un abastecimiento eficiente para las poblaciones locales. Esta situación exige el conocimiento detallado de las características hidrogeológicas de cada uno de estos pequeños acuíferos costeros.

Estudios geofísicos en superficie aplicados a la exploración de aguas subterráneas comenzaron a ser utilizados en la Península Nicoya a partir de la década de los setenta, son citados los trabajos de Protti (1981), Arias (com. esc., 2002); Arias et al. (2003) y Gómez (2005), entre otros.

En este trabajo fue utilizado el método de resistividad eléctrica con corriente continua para completar el modelado del acuífero de Playa Panamá. Este método permitió re-estimar los espesores de los sedimentos recientes, en donde el acuífero está albergado.

El área de estudio está circunscrito en una microcuenca costera de aproximadamente 20,2 km2, localizada en el límite norte de la Península de Nicoya. Playa Panamá constituye una de las playas que rodean Bahía Culebra (Fig. 1).

\section{MATERIALES Y MÉTODOS}

Para completar el modelo conceptual del acuífero costero fueron utilizados datos litoestrátigraficos de 12 pozos pertenecientes a los archivos del SENARA (2006) y del AyA (2006), los cuales fueron construidos en los depósitos recientes. Los espesores registrados en estos pozos fueron correlacionados con la nueva información obtenida a

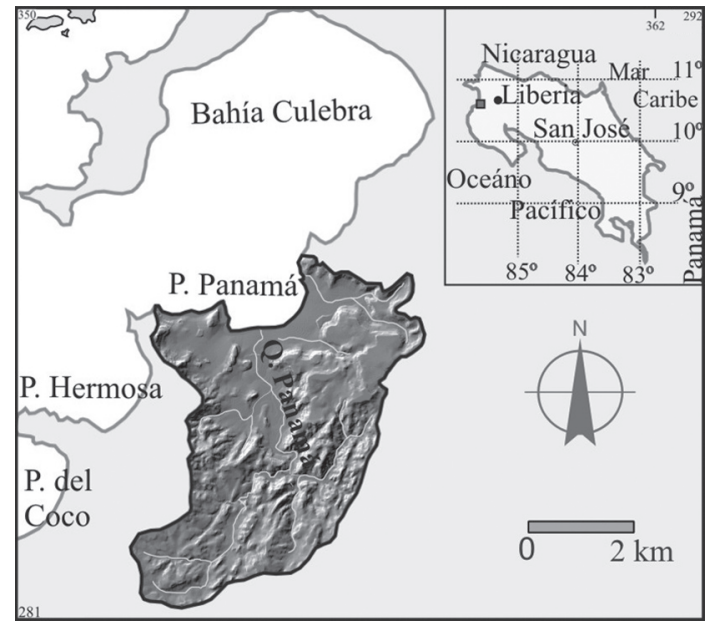

Fig. 1: Mapa de localización del área de estudio

partir de 12 Sondeos Eléctricos Verticales (SEVs). En la realización de los SEVs fue aplicado el dispositivo electródico tipo Schlumberger (Fig. 2), empleando una abertura máxima de $\mathrm{AB} / 2$ igual a $100 \mathrm{~m}$. El equipo usado fue el modelo Terrameter SAS 1000 de la ABEM ${ }^{\circledR}$ con cuatro electrodos y con una potencia máxima de $400 \mathrm{~V}$.

Los espesores de los sedimentos recientes fueron calculados por inversión de la resistividad aparente, se asumió un modelo inicial de cuatro capas: 1) el suelo superficial; 2) la zona no saturada; 3) la zona saturada y 4) el basamento regional, éste último conformado por el Complejo de Nicoya.

Los resultados fueron integrados mediante un Sistema de Información Geográfica (SIG), luego, los valores de espesor obtenidos de los registros de pozos y de los ensayos geofísicos fueron interpolados por medio del método Krigging para generar el mapa de isopacas.

\section{Método de resistividad eléctrica con corriente continua}

El uso de métodos resistivos con corriente continua (CC) aplicados a estudios de aguas subterráneas es bien conocido. La estimación de la resistividad del subsuelo $(\rho)$ a partir de su resistividad aparente se utiliza como un instrumento para evaluar los espesores de capas geológicas, las profundidades de niveles freáticos, e incluso 


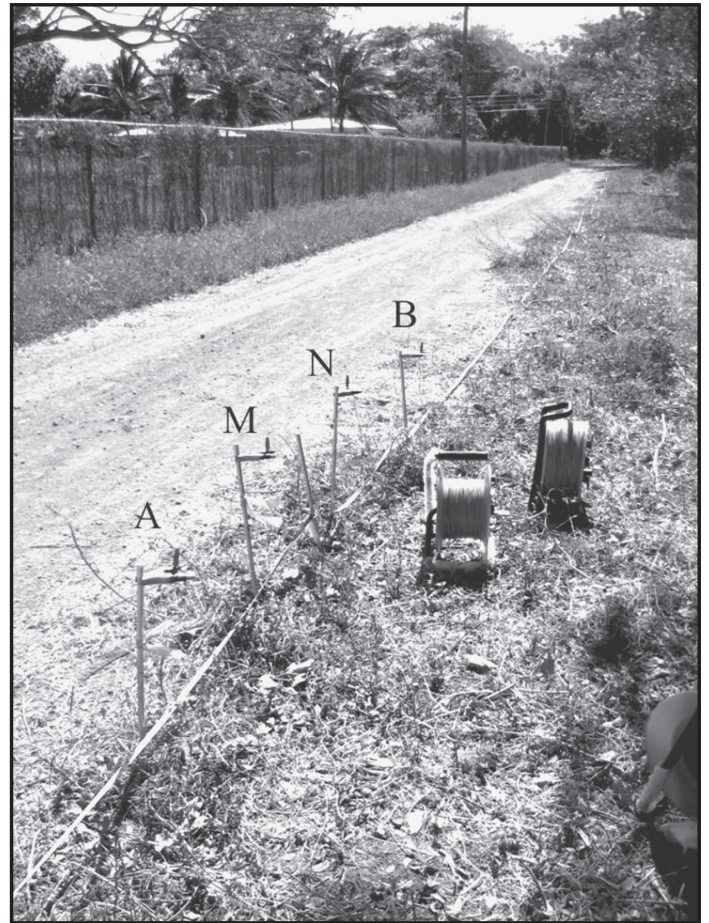

Fig 2: Arreglo electródico Schlumberger

las zonas de dispersión salina, entre otros. Ward (1990) afirma que en los acuíferos homogéneos con presencia de arcilla, la resistividad total del depósito es afectada por la resistividad de los poros del fluido, la porosidad de la formación rocosa, el grado de saturación, el volumen de la fracción arcillosa y las resistividades de las fracciones rocosa y arcillosa

En este trabajo, el acuífero de Playa Panamá fue considerado como heterogéneo, con presencia de arcillas, compuesto principalmente de arenas gruesas y guijarros. En este caso es difícil cuantificar, por separado, el volumen y la resistividad de la fracción arcillosa a partir de los datos de resistividad aparente del cuerpo total de sedimentos. Por tal razón, las resistividades medidas en los sedimentos del acuífero representan, básicamente, contenidos relativos de materiales de texturas granulométricas diferentes.

El método de resistividad eléctrica utiliza una fuente generadora de corriente (I), que es inyectada en el suelo a través de dos electrodos (A y B), la corriente produce una diferencia de potencial
(V) que es medida en los electrodos de potencial $(\mathrm{M}$ y N). La disposición relativa de los electrodos es conocida también como arreglo geoeléctrico y su escogencia depende de las características del objeto de estudio. En aplicaciones hidrogeológicas este método geofísico es utilizado en las técnicas de SEVs y barridos dipolares, los primeros permiten medir la variación vertical de la resistividad aparente debajo de un punto único, y son usados para estimar la posición y espesor de capas litológicas horizontales en profundidad. La segunda técnica permite obtener un perfil a lo largo de una línea de puntos, tiene la ventaja de analizar la variación lateral y en profundidad de la resistividad eléctrica del cuerpo geológico estudiado. La profundidad de investigación teórica máxima alcanzada en este estudio mediante la utilización del método fue de $100 \mathrm{~m}$, la cual, además es proporcional a la abertura de los electrodos A y B.

El conocimiento de la resistividad de la primera capa permite analizar la resistividad de los horizontes inferiores, pues si la capa superior mostrara valores muy bajos, los valores de los demás horizontes pueden ser subestimados. En este estudio, la resistividad del basamento se consideró como relativa, y no es representativa del tipo de roca porque el espesor total de esta unidad geológica es superior al alcance de la corriente eléctrica aplicada. Para evaluar la consistencia de la inversión de la resistividad del basamento, se consideró que los valores de resistividad aparente en los gráficos versus $\mathrm{AB} / 2$ debían estar dispuestos, aproximadamente, a lo largo de una recta con inclinación de $45^{\circ}$ con respecto a la horizontal.

\section{CONTEXTO GEOLÓGICO DELACUÍFERO}

Desde la perspectiva geotectónica, el acuífero de Playa Panamá pertenece a una cuenca hidrográfica situada en el margen noroeste del antearco volcánico, el cual está asociado a la zona de subducción formada por la interacción entre las placas tectónicas Cocos y Caribe. Este tipo de cuencas poseen áreas pequeñas con depósitos sedimentarios recientes, acumulados a partir de la depositación de material aluvial erosionado y transportado 
desde las sierras del antearco, así como material proveniente de la dinámica costera.

En el área estudiada existen tres conjuntos litológicos (Fig. 3). En la base de la secuencia estratigráfica está el Complejo de Nicoya, el cual está constituido por basaltos cretácicos y radiolaritas de edad Jurásico-Cretácico Inferior, así como por cuerpos intrusivos de edad Santoniano (Denyer \& Baumgartner, 2006). En esta área, el Complejo de Nicoya aflora entre las cotas de 0 y $170 \mathrm{~m}$ de altitud, en los sectores suroeste y noroeste de la microcuenca. La meteorización de los basaltos forma suelos arcillosos con espesores entre 1 y 2 $\mathrm{m}$, además estas rocas presentan fracturamiento considerable debido al tectonismo. Las radiolaritas no afloran en la microcuenca. El Intrusivo Potrero (miembro del Complejo de Nicoya), aflora en el extremo oeste de la faja costera de Playa Panamá. Denyer \& Arias (1993) afirman que esta subunidad intrusiva puede ser distinguida de los basaltos por su meteorización tipo "arenitizada".

El contacto superior del Complejo de Nicoya está representado por la Formación Bagaces, de edad Mioceno Superior-Plioceno (Gillot et al., 1994). Esta unidad geológica está constituida por lavas ácidas, depósitos lacustres, conglomerados y principalmente por ignimbritas, cuya manifestación en superficie $(2,4 \mathrm{~km} 2)$ se observa en el sector noreste del área de estudio. Estas rocas poseen un espesor de aproximadamente $30 \mathrm{~m}$.

Rasgos estructurales son identificables en la parte sur de la cuenca hidrográfica (Fig. 3). Alineamientos geológicos con dirección NE-SW y E-W fueron fotointerpretados, los cuales son asociados por Denyer \& Arias (1993) con un basculamiento regional del Complejo de Nicoya. Sin embargo, uno de esos alineamientos corresponde con una falla sinestral, cuyo sentido de movimiento fue interpretado a partir de estrías. Hidrogeológicamente los basaltos representan una unidad acuífera secundaria, y su potencial hídrico podría ser mayor a lo largo de estas zonas de fractura.

En la parte superior de la secuencia cronoestratigráfica están los depósitos recientes, separados, por su génesis, en depósitos costeros (nomenclatura de Angulo, 1993) y depósitos continentales. Los primeros están concentrados a lo largo de la faja costera y forman pequeños cordo- nes dunares de arena, distribuidos en la zona de pos-playa (nomenclatura de Bigarella et al., 1966) que abarca los sedimentos de berma; su extensión es entre 20 y $40 \mathrm{~m}$ y su espesor entre 1 y $3 \mathrm{~m}$. Los depósitos costeros incluyen también el material de textura limo-arcillosa formado en el estero (manguezal) de la desembocadura de la Quebrada Panamá. Los depósitos continentales conforman el acuífero libre poroso, el cual se extiende en una superficie de 3,9 km2 y está constituido principalmente por conglomerados angulares (nomenclatura de Fritz, 1988) monomícticos, intercalados con materiales de textura más fina. Todos sus componentes se derivan de las unidades geológicas supracitadas. El espesor de estos sedimentos es variable y alcanza hasta $27,4 \mathrm{~m}$. Los registros litológicos de los pozos revelaron la existencia de una capa arcillosa en la parte superior del acuífero (Fig.4). AyA (2006) señala que el acuífero mostró cierta condición de semi-confinamiento en el sector $\mathrm{E}$ de los depósitos recientes, con base en el ascenso observado del nivel freático después de la perforación del pozo CN-336. Esta situación podría estar asociada con la presencia de la capa arcillosa, cuyo espesor medio en el sector central de los depósitos recientes es de aproximadamente 6 m., sin embargo, el mismo no seria constante en toda el área que abarcan los depósitos. La sección central de los depósitos continentales está formada por intercalaciones decimétricas de bloques y guijarros poco redondeados, además muestran acumulaciones lenticulares de arcilla y formas sedimentarias del tipo laminación paralela y laminación cruzada. Todas estas características reflejan poco transporte del material, depositado en un ambiente de alta energía.

\section{MODELO CONCEPTUAL DEL ACUÍFERO A PARTIR DE DATOS GEOFÍSICOS}

El perfil geológico general indicado por los registros litológicos de pozos fue sintetizado en tres horizontes. En la parte superior estaría el suelo superficial que es poco desarrollado y está formado principalmente por material de textura arcillosa, la parte central corresponde con el acuífero y por último está el basamento 


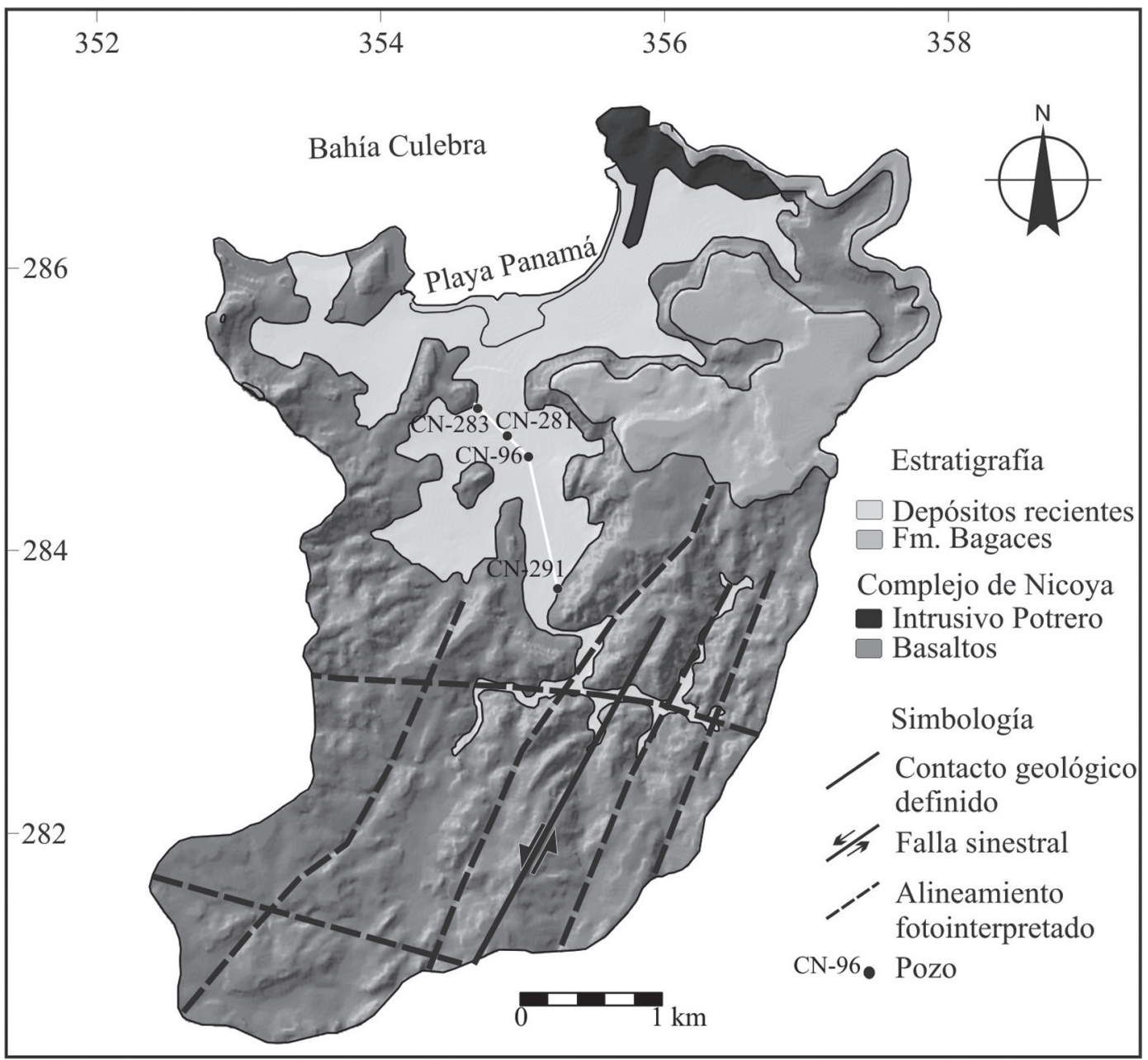

Fig. 3: Mapa geológico de la cuenca hidrográfica de Playa Panamá. La línea blanca marca el perfil presentado en la Fig. 3

basáltico. En el modelo asumido, los sedimentos fueron subdivididos en zona saturada (ZS) y zona no saturada (ZNS), cuyo límite está representado por el nivel freático. La distribución de los pozos principales y los SEVs ejecutados en la zona acuífera es mostrada en la Fig. 5.

Los SEVs 01 a 06 fueron realizados en los sectores sur y central de la zona acuífera. Las curvas de inversión de resistividad aparente fueron muy similares en cinco de ellos, las diferencias obedecen a la variación en los espesores de las capas asumidas en el modelo (Fig.6). Las curvas con concavidad más acentuada sugieren profundidades mayores del techo del basamento, el cual está representado en el tramo final ascendente de las mismas.

El SEV01 fue realizado sobre los sedimentos arenosos del lecho de la Quebrada Panamá. La resistividad superficial medida en este SEV (500 $\Omega$ $\mathrm{m})$ está relaciona con la mayor porosidad y menor compactación del material arenoso, la ZNS tendría una mezcla de este material con arcilla, pues su resistividad fue de $60 \Omega \mathrm{m}$. El nivel freático fue identificado a partir de $8 \mathrm{~m}$ de profundidad, puesto que en este horizonte el valor de resistividad disminuyó a $30 \Omega \mathrm{m}$ debido al incremento relativo de la conductividad eléctrica originado por la presencia del agua. Es probable que los valores 


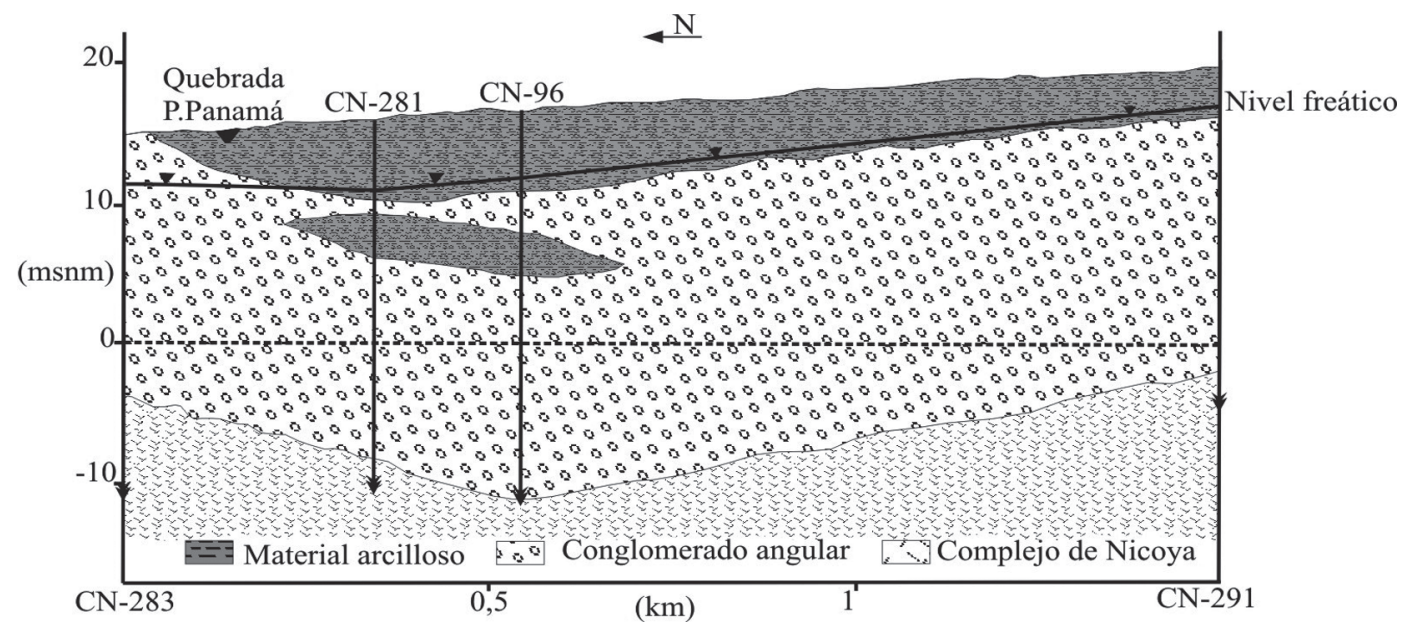

Fig.4: Perfil estratigráfico de la sección central de la zona acuífera de Playa Panamá

de resistividad de la ZS y de la ZNS fueran afectadas por la alta resistividad de las arenas secas de aluvión. El error de inversión de este SEV (16 \%) fue relacionado con una dispersión mayor de los datos, producto de la no linealidad del sondeo por causa de las condiciones geomorfológicas en ese sitio (curva en el cauce).

El SEV03 ubicado también en el sector sur de la zona acuífera mostró resultados diferentes al resto de los cinco SEVs iniciales. La primera capa además de poseer algo de arena, exhibiría mucho más arcilla y una disminución en su porosidad efectiva. Este hecho fue reflejado en su resistividad de $32 \Omega \mathrm{m}$. El valor de resistividad en la ZNS (52 $\Omega \mathrm{m})$ sugirió que el contenido de arcillas es menor y el material predominante seria de textura gruesa (conglomerados angulares). La presencia de los conglomerados en la ZS controlaría, por dispersión de la corriente eléctrica (porosidad mayor), el valor de la resistividad en la ZS (42 $\Omega \mathrm{m})$. Este SEV registró el mayor espesor calculado de depósitos recientes $(\sim 25 \mathrm{~m})$.

Los SEVs 02, 04, 05 y 06 revelaron condiciones similares en el contexto geológico de subsuelo. El suelo es arcilloso y su resistividad osciló entre 32 y $45 \Omega \mathrm{m}$. Sin embargo, la resistividad de la ZNS fue menor (10 a $18 \Omega \mathrm{m})$, lo cual sugirió que la compactación y el grado de saturación del material (principalmente arcillas) deben ser mayores en esos sectores. La ZS exhibió valores menores de resistividad $(7,5$ a $12 \Omega m)$ con relación a las capas superiores. $\mathrm{Si}$ bien el material está compuesto esencialmente por los conglomerados, la presencia de material arcilloso y del agua a una profundidad menor condicionó sus propiedades resistivas.

El perfil geolectrico de los sectores central y sur del acuífero muestra las variaciones en la resistividad eléctrica de los materiales y su correlación geológica interpretada (Fig. 7).

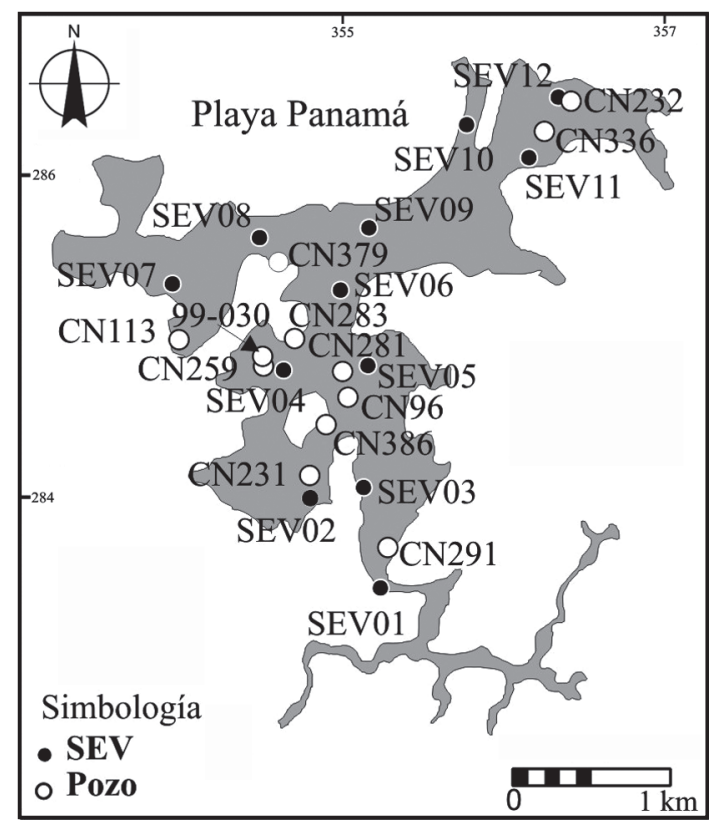

Fig 5: Localización de pozos y SEVs en el área de estudio 


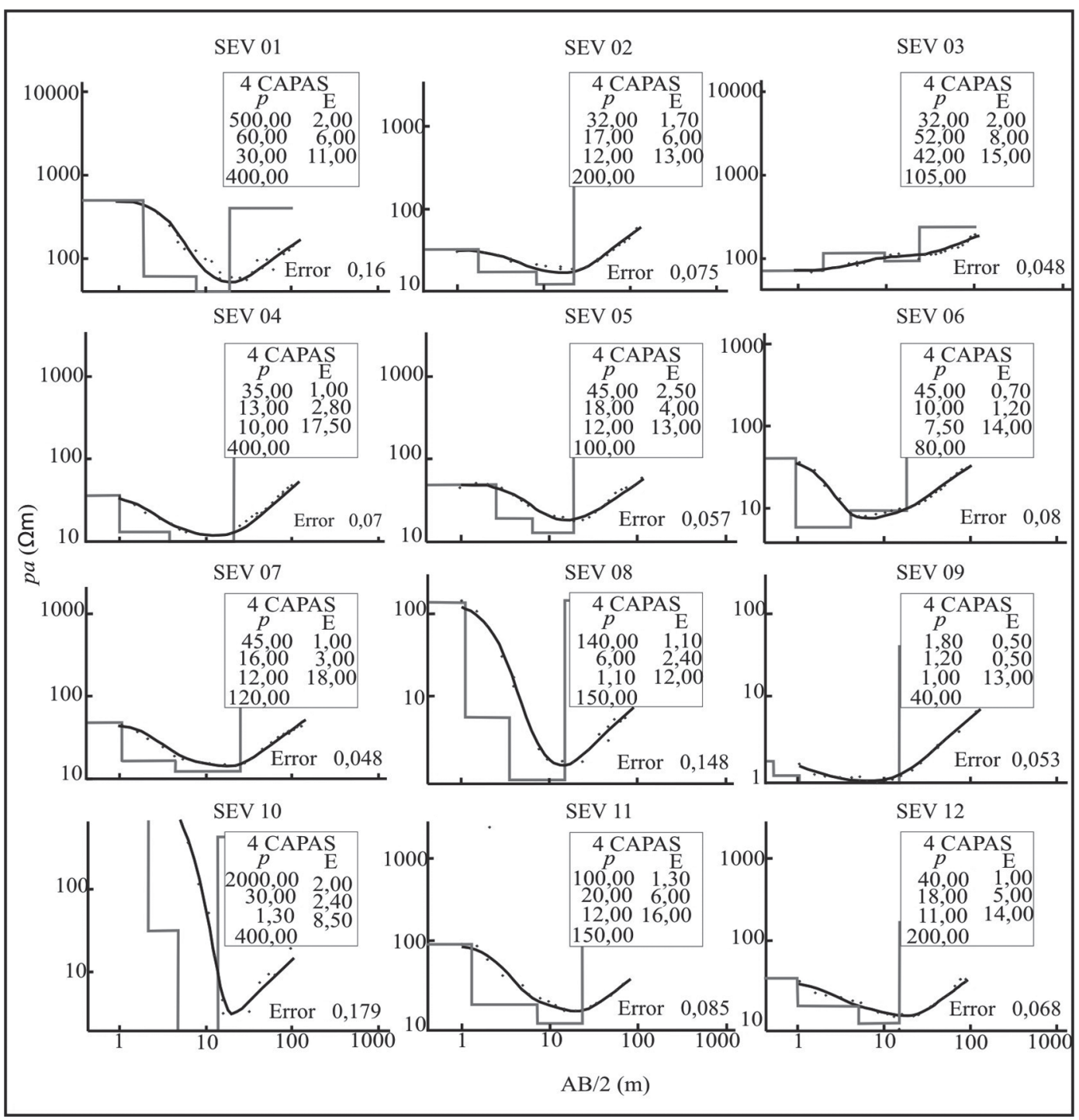

Fig. 6: Resultados de las inversiones de resistividad aparente medida en los SEVs

En el sector noroeste de los depósitos recientes el perfil de resistividad del modelo propuesto fue muy semejante al del sector central (SEVs 02 , 04,05 y 06). Los resultados de la inversión del SEV07 mostraron que el espesor de los depósitos en este sector es de aproximadamente $22 \mathrm{~m}$.

El resultado de la inversión de los SEVs 11 y 12 , efectuados en el sector noreste no mostró variaciones significativas en la resistividad de las capas asumidas en el modelo. El valor de resistividad del suelo superficial del SEV11 $(100 \Omega \mathrm{m})$ obedece a su localización sobre un relleno, de aproximadamente
$1,5 \mathrm{~m}$ de espesor, utilizado en la construcción de la calle local, factor que influyó en la porosidad y compactación del material. La ZNS y la ZS presentaron resistividades promedio de 19 y $11,5 \Omega \mathrm{m}$ respectivamente, lo cual indica que el material predominante es de textura arcillosa.

Los SEVs 08 a 10, localizados en la faja costera, mostraron inversiones muy diferentes al resto de SEVs. Es importante mencionar que el SEV09 fue realizado sobre los sedimentos finos del estero Panamá. Los SEVs 08 y 10 fueron ejecutados sobre la zona de berma en los depósitos 


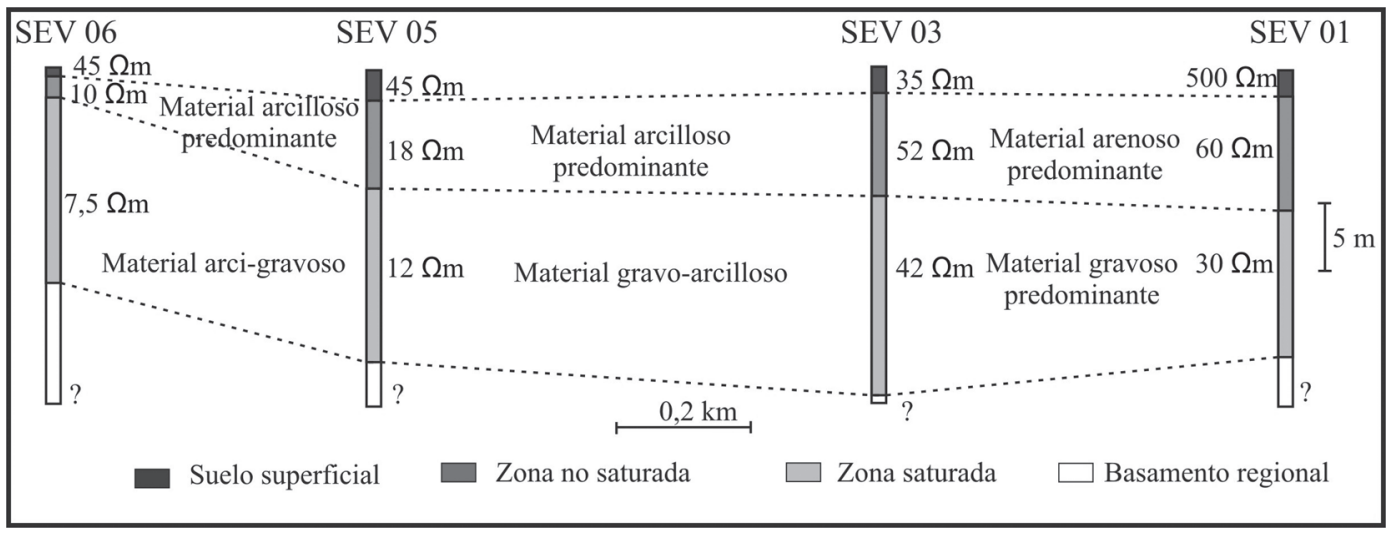

Fig. 7: Perfil geoeléctrico de la sección principal del acuífero.

costeros. En el SEV09 es evidente la influencia de la baja resistividad (o alta conductividad) de las arcillas en los resultados de la inversión, tanto para la el suelo superficial como para la ZNS. La resistividad eléctrica en estos horizontes del sondeo no superó el valor de 1,8 $\Omega$ m., lo cual es muy bajo en relación con los SEVs realizados después de la faja costera. Este sondeo también sugirió la presencia e influencia de la cuña salina en la ZS $(1 \Omega \mathrm{m})$, pues de acuerdo con Wilson et al. (2006), la conductividad del agua de mar es de aproximadamente $30 \mathrm{mhos} / \mathrm{m}(0,3 \Omega \mathrm{m})$; un depósito de sedimentos sin presencia de arcilla, saturado con agua salada, tendría una resistividad entre 5 e $10 \Omega \mathrm{m}$.

En los SEV s 08 y 10, el suelo superficial presentó valores de resistividad elevados (140 y 2000 $\Omega \mathrm{m})$ debido a la alta porosidad efectiva de las arenas secas. Sin embargo, el contraste con la resistividad de la ZS es evidente, pues los valores entre 1,1 e 1,3 $\Omega \mathrm{m}$ indican también de la presencia de la zona de dispersión salina a una profundidad inferior a 15,5 m (espesor máximo de los sedimentos en esos sondeos).

Los resultados de la inversión de los SEVs ubicados en la faja costera revelaron también que el espesor del acuífero diminuye en dirección al mar (Figs. 6 y 7).

Para verificar la equivalencia de los resultados de las inversiones de los SEVs realizados, los espesores calculados a partir de éstos fueron comparados con los espesores registrados en algunos pozos (cuadro 1).

La diferencia entre los espesores calculados a partir de los resultados de inversión de los SEVs y los obtenidos a partir de registros de los pozos fue poco significativa en la mayoría de los casos considerados. Es preciso aclarar que el error en la inversión no significa error en el modelo. Para el SEV01, la diferencia elevada fue causada por la dispersión de los datos de resistividad aparente en la curva de inversión, debido a las condiciones geológicas y geomorfológicas del sitio donde fue ejecutado el sondeo. En el SEV12, el contraste obedecería a una ambigüedad en la descripción litológica del pozo CN-232 (registro de 1989), según el cual, habría basalto meteorizado entre las profundidades de 7 y $14 \mathrm{~m}$. Sin embargo, de acuerdo con los valores de resistividad eléctrica calculados en la ZNS $(18 \Omega \mathrm{m})$ y en la ZS $(11 \Omega \mathrm{m})$, con el error de la inversión en el SEV12 y con la descripción del pozo más cercano ( $\mathrm{CN}-336)$, esos horizontes son correlacionables con los conglomerados angulares (con predominancia de material arcilloso). Así, el espesor total de los depósitos recientes en este sondeo seria de $21 \mathrm{~m}$ y no de $7 \mathrm{~m}$. En tal caso, la diferencia de correlación entre ambos espesores disminuiría a $5 \%$. Otro factor que influyó en los valores de las diferencias de correlación fue la distancia de separación entre los pozos y los SEVs (cuadro 1), pues la profundidad del basamento puede variar significativamente de un lugar a otro debido a las variaciones paleotopográficas de los basaltos.

El modelo conceptual del acuífero implica la existencia de tres fronteras principales, dos de ellas son móviles y están representadas por el nivel freático en límite superior y por la zona de dispersión salina en el límite norte. Los límites 


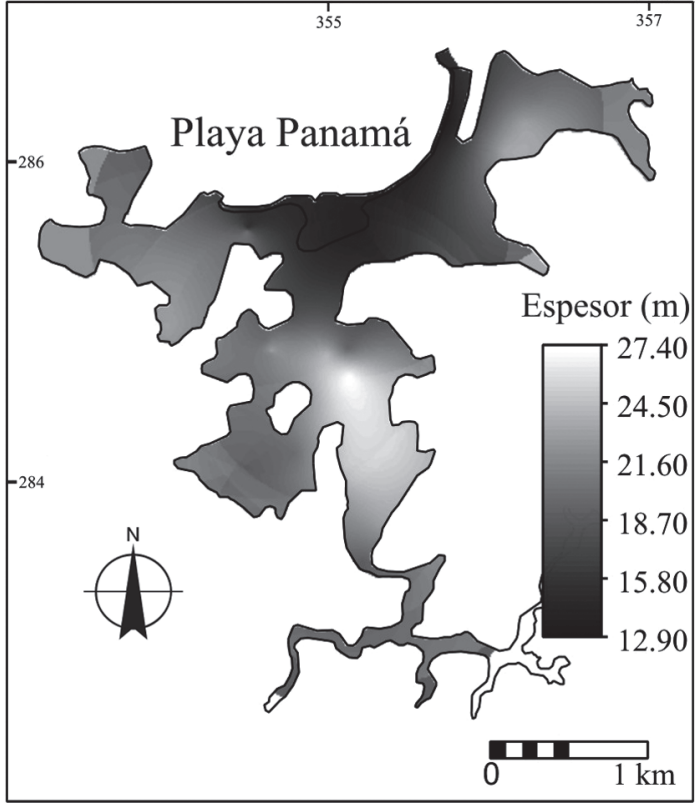

Fig. 8: Mapa de espesores de los depósitos recientes. El sector sur muestra dos áreas que no fueron abarcadas por la interpolación realizada

laterales e inferiores corresponden con el contacto de los sedimentos con el basamento regional, este último controla la geometría y la distribución del espesor del acuífero. En la Fig. 8 es presentado el mapa de isopacas para la zona acuífera, el cual incluye los datos de los 12 SEVs y los 12 pozos. El sector central presenta el espesor mayor de sedimentos y corresponde con una depresión paleogeográfica, posiblemente un paleovalle asociado con la topografía original de los basaltos del Complejo de Nicoya.

\section{CONSIDERACIONES FINALES}

Las características hidrogeológicas del acuífero están condicionadas grandemente por el contexto geológico y geotectónico en la cuenca hidrográfica de Playa Panamá. Exhibe una homogeneidad composicional dominada por la presencia de los basaltos del Complejo de Nicoya, y está caracterizado por una heterogeneidad textural originada por una ciclicidad en las condiciones de depositación de los materiales continentales. La distribución de espesores en los depósitos que integran el acuífero libre de Playa Panamá está restringida por la paleotopografía moldeada sobre los basaltos. Acuíferos secundarios, particularmente en el Complejo de Nicoya, tendrían mayor potencial hídrico a lo largo de los rasgos estructurales regionales.

La inversión de la resistividad aparente en cada SEV partió del modelo conceptual inicial con cuatro horizontes, el cual fue considerado válido porque refleja las características litológicas registradas en los pozos y las observadas en los

Cuadro 1

Comparación de los resultados calculados a partir de los SEV con algunos pozos de la zona acuífera.

\begin{tabular}{|c|c|c|c|c|c|}
\hline & $\begin{array}{c}\text { Separación rela- } \\
\text { tiva }(\mathrm{m})\end{array}$ & $\rho(\Omega \mathrm{m})$ & Espesor (m) & $\begin{array}{l}\text { Diferencia de la } \\
\text { correlación }(\%)\end{array}$ & $\begin{array}{c}\text { Error de la } \\
\text { inversión }(\%)\end{array}$ \\
\hline SEV01 & 255 & $30-500$ & 19 & 17 & 16,3 \\
\hline Pozo CN-291 (SENARA) & & & 23 & & \\
\hline SEV02 & 140 & $12-32$ & 20,7 & 3,5 & 7,5 \\
\hline Pozo CN-231 (SENARA) & & & 20 & & \\
\hline SEV04 & 120 & $10-35$ & 21,3 & 7,3 & 7,0 \\
\hline Pozo CN-259 (SENARA) & & & 23 & & \\
\hline SEV07 & 340 & $12-45$ & 22 & 4,3 & 4,8 \\
\hline Pozo CN-113 (SENARA) & & & 23 & & \\
\hline SEV11 & 190 & $12-100$ & 23,3 & 1,3 & 8,5 \\
\hline Pozo CN-336 (AyA) & & & 23 & & \\
\hline SEV12 & 80 & $11-40$ & 20 & 65 & 6,8 \\
\hline Pozo CN-232 (AyA) & & & 7 & & \\
\hline
\end{tabular}


afloramientos en el campo. La separación del suelo superficial de la ZNS fue sustentada en que el horizonte inicial presenta diferencias significativas de composición física, química y biológica.

Los resultados de los ensayos de resistividad eléctrica con corriente continua no permitieron estimar un valor representativo de resistividad para cada tipo de material. Sin embargo, los valores relativos de resistividad ayudaron a identificar el predominio de unos materiales sobre otros. En el sector sur de la zona acuífera los materiales serian de textura gruesa (arena y gravas). El sector central estaría caracterizado por una distribución textural de materiales más equilibrada, pero con predominancia de arcillas en la parte superior, los sectores noroeste y noreste, con excepción de las áreas de la faja costera, mostrarían condiciones geológicas de subsuelo similares a este sector. El sector norte exhibe predominio de materiales de textura arcillosa; el material de textura gruesa yacería básicamente en la parte inferior de los depósitos.

Los SEVs ofrecieron resultados satisfactorios en la determinación de los espesores de los horizontes estudiados y concedieron un mejor entendimiento de la estratigrafía del subsuelo. El espesor de la zona acuífera es menor en la zona costera que en las áreas del interior del continente, el sector central posee el espesor mayor $(27,4$ $\mathrm{m})$. Este rasgo geométrico del reservorio hídrico poroso influiría en el control de la hidrodinámica subterránea, particularmente en la intensidad del proceso de intrusión salina. La existencia de una única zona de dispersión salina en el acuífero de Playa Panamá estaría sustentada en su simplicidad hidrogeológica (tipo libre). Sin embargo, Custodio (1987) manifiesta que la existencia de una única zona de dispersión se dará cuando ella represente poco porcentaje del espesor saturado, situación que no fue reflejada en los resultados de las inversiones geofísicas efectuadas. En los SEVs 08 al 10 el espesor saturado de sedimentos representa un porcentaje superior al $65 \%$.

\section{AGRADECIMIENTOS}

El autor agradece a ALHSUD, por la ayuda financiera durante los estudios de posgrado. Al personal del AyA de la sedes de Liberia y Central, especialmente a Grace García. A los profesores Dr. Ernani Francisco da Rosa Filho, Dr. Augustinho Rigoti y Dr. Francisco Ferreira. Al personal de los laboratorios de Pesquisas Hidrogeológicas (LPH) y Pesquisas en Geofísica Aplicada (LPGA) de la Universidade Federal do Paraná (UFPR). A los siguientes colegas geólogos por sus contribuciones en el trabajo de campo: Natalia Montes, Esteban Bonilla, Pablo Herrera, Natalia Zamora, Pedro Acosta, Alex Padilla, Catalina Vargas, Gelu López y a Oscar Lucke. Al personal de la Escuela Centroamericana de Geología (ECGCR), particularmente al Prof. Dr. Mario Arias por el préstamo del equipo de geofísica utilizado. A los árbitros revisores Carlos Leandro y Sergio Mora, por sus sugerencias.

\section{REFERENCIAS}

ÂNGULO, R., 1993: Morfologia e gênese das dunas frontais do litoral do estado do Paraná. - Revista Brasileira de Geociências, 23: 68-80.

ARELLANO,F.\& VARGAS,A., 2001: Casos de Contaminación por intrusión salina en acuíferos costeros de la Península de Nicoya (Costa Rica). - Rev. Geol. Amér. Central, 25:77-84.

ARIAS SALGUERO, M., VARGAS, A. \& GUÈRIN, R. 2003: Geofísica aplicada al problema de la intrusión salina en los acuíferos costeros de Costa Rica. - En: IGME (ed): Tecnología de la intrusión de agua de mar en acuíferos costeros: países mediterráneos, Madrid, 8 (1): 163-167.

AyA, 2006: Inventario de pozos, hoja topográfica Carillo Norte.- Escala 1:50 000, IGN, San José. 
BIGARELLA, J.J., FREIRE, S.S., SALAMUNI, R. \& VIANA, R., 1966: Contribuição ao estudo dos sedimentos praiais recentes, II praias de Matinhos e Caiobá. - Boletim da Universidade Federal do Paraná Geografia Física, 6.- 109 págs.

CUSTODIO,E., 1987: Salt-fresh water interrelationships under natural conditions. En: CUSTÓDIO, E. \& BRUGGEMAN G.A. (eds): Groundwater problems in coastal areas. - Studies and reports in hydrogeology. Bélgica, UNESCO. 14-88.

DENYER, P. \& ARIAS, O., 1993: Geología del Norte de la Península de Nicoya. - Rev. Geol. Amér. Central, 16: 69-84.

DENYER, P. \& BAUMGARTNER, P.O., 2006: Emplacement of Jurassic-Lower Cretaceus radiolaritas of the Nicoya Complex. - Geologica acta, 4: 203-208.

FRITZ, W.J., 1988: Basics of physical stratigraphy and sedimentology.- 371 págs. J. Wiley \& Sons, New York.

GÓMEZ, M. 2005: Estudio hidrogeológico Integral de las cuencas hidrográficas de los poblados el Coco y Ocotal, Carrillo, Guanacaste. - 168 págs. Univ. de Costa Rica, San José, [Tesis M.Sc.].
GILLOT P.Y., CHIESA, S.\& ALVARADO, G., 1994: Cronostratigraphy of upper MioceneQuaternary vulcanism in northern Costa Rica. Rev. Geol. Amér. Central, 17: 45-53.

LOSILLA, M. 1993. Evidencia de amenaza a las aguas subterráneas de Costa Rica. - 23 págs. Taller de aguas subterráneas, Colegio Federado de Ingenieros, San José.

PROTTI, R. 1981: Geología y geofísica con fines hidrogeológicos en la planicie costera Jicaral-Santa Rita, Península de Nicoya. - 47 págs. + 5 mapas. Univ. de Costa Rica, San José, [Tesis Lic.].

SENARA, 2006: Inventario de pozos hoja topográfica Carrillo Norte.- Escala 1:50 000, IGN, San José.

WARD, S.H., 1990: Resistivity and induced polarization methods. - En: Ward, S.H (ed): Geotechnical and Environmental Geophysics. Investigations in Geophysics, SEG, 5 (1): 147190.

WILSON, S.R., INGHAM, M. \& McCONCHIE, J.A., 2006: The applicability of earth resistivity methods for saline interface definitions. - J. Hidrol. 316: 301-312. 